\title{
IDENTIFICATION OF HEREDITY KERNELS AND THEIR INFLUENCE ON THE LIFE TIME OF GLASS/POLYESTER COMPOSITES
}

\author{
E.T. OLODO* \\ EPAC/Department of Civil Engineering \\ University of Abomey-Calavi (UAC), BENIN \\ 03BP1409 Cotonou (Benin) \\ E-mail: olodoe@live.fr \\ E.C. ADJOVI \\ EPAC/Department of Civil Engineering \\ University of Abomey-Calavi (UAC), BENIN \\ V. ADANHOUNME \\ International Chair of Mathematical Physics and Applications \\ (ICMPA-UNESCO Chair), University of Abomey-Calavi (UAC), BENIN
}

\begin{abstract}
One of the major problems encountered in prediction of hereditary viscoelastic behavior of polymeric composites is the determination of heredity kernels. This issue comes down to identification of the model characterizing the viscoelastic properties of these materials. The purpose of this work is to propose a model for prediction of viscoelastic nonlinear behavior of laminate composite with polyester matrix, through the study and analysis of heredity kernels and their influence on the life time of this material. Identification of this model required experimental determination at room temperature, of viscoelastic parameters of heredity kernels by macroscopic approach. These data provide predictive tools for establishment of the life time and long term stress limit under static complex loading for this type of material.
\end{abstract}

Key words: heredity kernels, viscoelastic parameters, life time.

\section{Introduction}

Polymeric composites frequently share the nature of resins in their manufacture, present linear or non-linear viscoelastic properties. In particular, some fiberglass polymeric composites behaviour becomes non-linear before reaching strains corresponding to normal use stresses (Schapery, 2000; Zhang et al., 1992; Brinson et al., 1986). The principle of superposition of Boltzman should be used (Pobedria, 2007). Factors such as temperature and the history of the stresses will considerably affect the life time (Sullivan, 1990; Augl, 1987; Suvorova, 2010).

The question of the determination of influence functions of non-linear viscoelastic models for polymeric fiberglass composites is only partially addressed in the technical literature and few models are proposed. These models are unfortunately not robust. One of the major difficulties encountered in the establishment of these models lies in the experimental identification of viscoelastic parameters of the material taking strictly into account the viscoelastic nonlinear behaviour thereof. On the other hand, one of the current problems in the study of composites is the prediction of their holding in long term, necessary for

\footnotetext{
* To whom correspondence should be addressed
} 
design of structures based on these materials. These theme-based approaches are based on models of damage and hereditary creep in which assessment of the long-term behaviour models must be established as integral operators (Schapery, 1997 and Dimitrienko, 1989). In these conditions, one of the critical issues is the determination of type of influence functions or operator kernels and their parameters. Experimental data on these issues are not much. The objective of this work is to study and analyze the possible influence of functions through the experimental determination of their parameters in order to assess their impact on the life time of fiber glass composites.

\section{Materiel and method}

The studied material is an orthotropic glass/polyester laminate composite 2D (Fig.1).

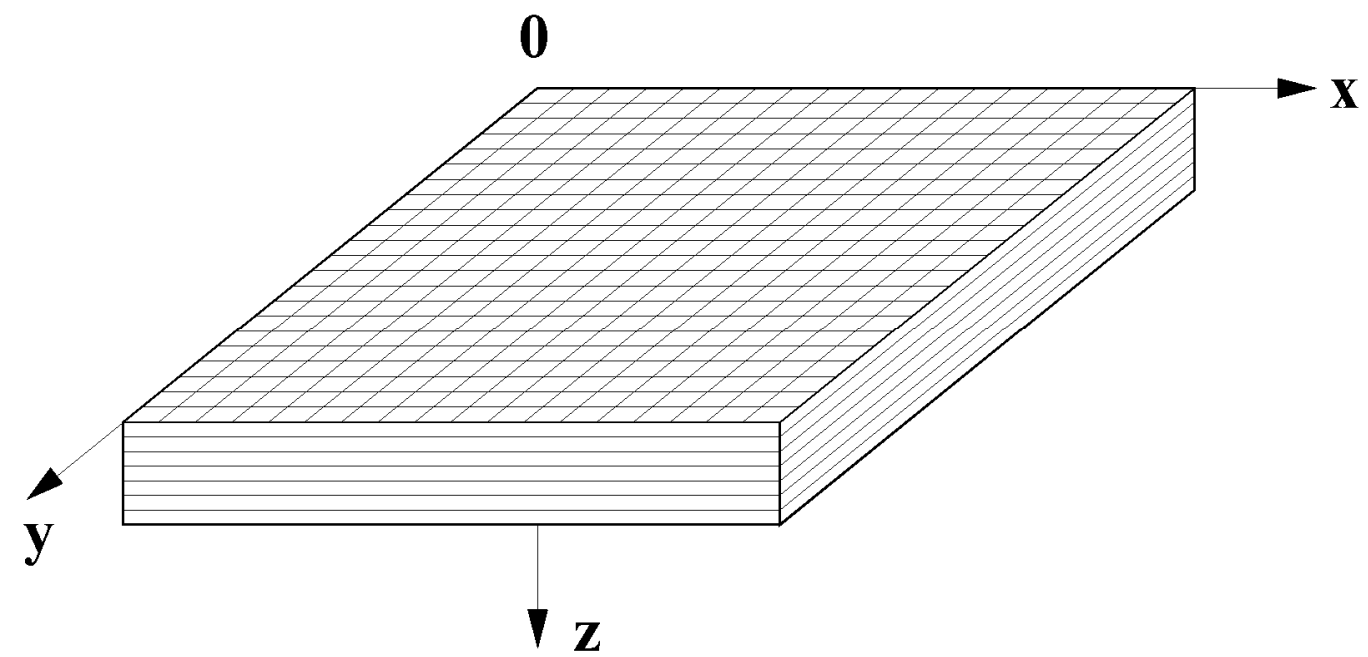

Fig.1. Laminate glass/polyester Composite.

Anisotropic nonlinear viscoelastic models are based mostly on damaged approaches. At the macroscopic scale, these approaches are similar to Goldenblat-Kopnov's long term criterion for anisotropic materials

$$
\left.\Phi(t)=\int_{0}^{t} \omega_{i k}(t-\xi) \sigma_{i k}(\xi) d \xi+\left[\int_{0}^{t} \int_{0}^{t} \omega_{i k m n}\left(t-\xi_{1}, t-\xi_{2}\right) \sigma_{i k}\left(\xi_{1}\right) \sigma_{m n}\left(t-\xi_{2}\right) d \xi_{1} d \xi_{2}\right)\right]^{\frac{1}{2}} \leq 1
$$

This non-linear model is based on coupling Mises-Hill plastic potential for anisotropic materials and A.A. Ilyushin kinetic approach, where:

$t$ - time after loading of the material,

$\xi$ - including time in interval $0 \leq \xi \leq t$,

$\sigma_{i k}(\xi), \sigma_{m n}(\xi)$ - Functionals setting program loading of the material;

Argument $(t-\xi)$ assumes invariance of functinals to loading time

$\omega_{i k}$ and $\omega_{i k m n}$ are the heredity kernels of the model proposed in the present work. 
Expression (2.1) assumes that the first full operator including the linear components of stress tensor is linear pulse input $\sigma_{i k}(\xi) d \xi$ and corresponding heredity kernel $\sigma_{i k}(t-\xi)$, on the total value of the damage $\phi(t)$.

Similarly, the second term of Eq.(2.1) is the joint influence of the two previous pulses $\sigma_{i k}\left(\xi_{l}\right) \mathrm{d} \xi_{l}$, $\sigma_{m n}\left(\xi_{2}\right) d \xi_{2}$ and the kernel $\omega_{i k m n}\left(t-\xi_{1}, t-\xi_{2}\right)$ on the total value of the damage.

The third approximation, in principle, is operated in similar manner on three previous time intervals, and so on. Therefore the global supply of pulses in the functional $\phi(t)$ would be the sum of the input linear, quadratic, cubic, etc.

Expression (2.1) is limited to linear and quadratic terms because this model assumes that the tensor heredity kernel should respectively be the second and fourth order that preserves the invariance of the model to the transformation groups.

Accordingly, the heredity kernels must meet the following conditions

1.

$$
\begin{aligned}
& \omega_{i k}(t, \xi)=\omega_{i k}(t-\xi), \\
& \omega_{i k m n}\left(t, \xi_{1}, \xi_{2}\right)=\omega_{i k m n}\left(t-\xi_{1}, t-\xi_{2}\right) .
\end{aligned}
$$

2. $\quad \frac{\partial \omega_{i k}(t, \xi)}{\partial \xi} \succ 0$

$$
\frac{\partial^{2} \omega_{i k m n}\left(t, \xi_{1}, \xi_{2}\right)}{\partial \xi_{1} \partial \xi_{2}} \succ 0 .
$$

The first condition means that the model must keep its invariance to the origin of the time;

The second condition means that with increasing of $\zeta$, the influence of the stresses occurring in the final moments, increases and effects the material damage rate.

The shape of the heredity kernel of the model must be specific to each material. Especially for the polymeric composites reinforced fiberglass a functional relationship must be established with the static resistance tensor (Pobedria, 1983)

$$
\begin{aligned}
& \omega_{i k}(t-\xi)=\prod_{i k} f_{1}(t-\xi), \\
& \omega_{i k m n}\left(t-\xi_{1}, t-\xi_{2}\right)=\prod_{i k m n} f_{2}\left(t-\xi_{1}, t-\xi_{2}\right)
\end{aligned}
$$

$\Pi_{i k}$ and $\Pi_{i k m n}$ are static resistance tensors, respectively, the second and fourth order and are determined in (Olodo et al., 2013); the functions $f_{1}$ and $f_{2}$ must be determined by tests of resistance in the long term.

For the studied laminate composite, we will consider the Abelian kernel

$$
\begin{aligned}
& \omega_{i k}(t-\xi)=\frac{a(1-\beta) \prod_{i k}}{(t-\xi)^{\beta}} \\
& \omega_{i k m n}\left(t-\xi_{1}, t-\xi_{2}\right)=\frac{a^{2}(1-\beta)^{2} \prod_{i k m n}}{\left(t-\xi_{1}\right)^{\beta}\left(t-\xi_{2}\right)^{\beta}} .
\end{aligned}
$$


Here, $\beta$ and $a$ are parameters to be determined by uniaxial testing in the long term;

$a$ is expressed in [time $]^{\beta}$

$\beta$ - without unit.

We will adopt a loading of the form

$$
\sigma_{i k}(\xi)=\sigma_{i k_{(0)}} \cdot \xi^{r}
$$

For a static loading, $r=0$, so

$$
\begin{aligned}
& \sigma_{i k}(\xi)=\sigma_{i k_{(0)}}, \\
& \sigma_{m n}(\xi)=\sigma_{m n_{(0)}} .
\end{aligned}
$$

Expression (2.1) then becomes

$$
\begin{aligned}
& \Phi(t)=\int_{0}^{t} \frac{a(1-\beta) \prod_{i k} \sigma_{i k(o)}}{(t-\xi)^{\beta}} d \xi+\left[\int_{0}^{t} \int_{0}^{t} \frac{a^{2}(1-\beta)^{2} \prod_{i k m n} \sigma_{i k(0)} \sigma_{m n(0)}}{\left(t-\xi_{1}\right)^{\beta}\left(t-\xi_{2}\right)^{\beta}} d \xi_{1} d \xi_{2}\right]^{0,5} \leq 1 \\
& a\left(\prod_{i k} \sigma_{i k(0)}+\sqrt{\prod_{i k m n} \sigma_{i k(0)} \sigma_{m n(0)}}\right) t^{1-\beta} \leq 1 .
\end{aligned}
$$

The state limit, $t$ becomes the time of failure $t_{*}$ of the material, so

$$
t_{*}=\left[a\left(\prod_{i k} \sigma_{i k(0)}+\sqrt{\prod_{i k m n} \sigma_{i k(0)} \sigma_{m n(0)}}\right)\right]^{\frac{1}{\beta-1}} .
$$

For uniaxial solicitation, expression (2.8) will be

$$
t_{*}=\left(\frac{a \sigma_{b i_{\bullet}}}{\sigma_{b}}\right)^{\frac{1}{\beta-1}}
$$

and the long term strength limit of the material will be

$$
\sigma_{b i *}=\frac{\sigma_{b}}{a t_{*}^{1-\beta}}
$$

$\sigma_{b}$ is the material resistance limit in uniaxial solicitation.

Expression (2.8) represents the life time of the material under static solicitation.

In this expression the part in parentheses is known, the determination of the parameters $a$ and $\beta$ of the heredity kernels is necessary to the life time prediction for this type of loading. 


\section{Results and discussion}

\subsection{Description of the test}

The parameters $a$ and $\beta$ are determined by long term uniaxial compression tests. To do this, experimental device for deformation testing is used. This device is equipped with an electronic clock to set the time corresponding to a given stress level including the break time of the test piece (Fig.2). The tests are performed on tubular composite tubes reinforced with glass fiber (Fig.3).

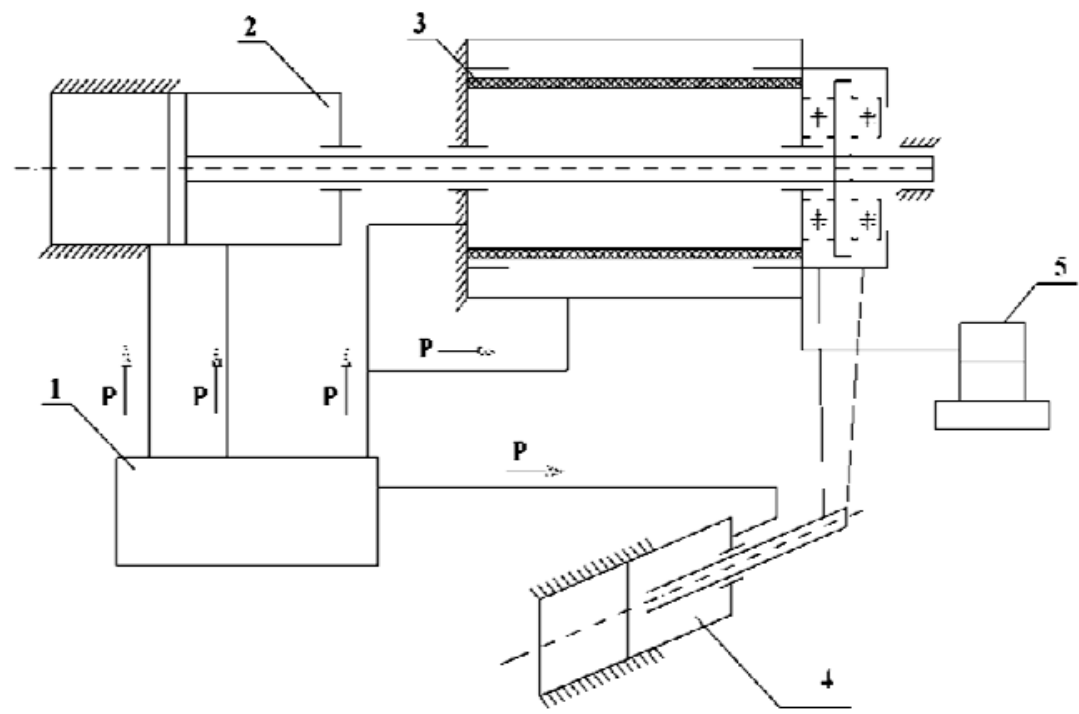

Legend:

$1,2,4$ - loading system of the test piece

3 -test tube

5 -electronic lock and data logger

Fig.2. Experimental long term testing device on glass/polyester composite.

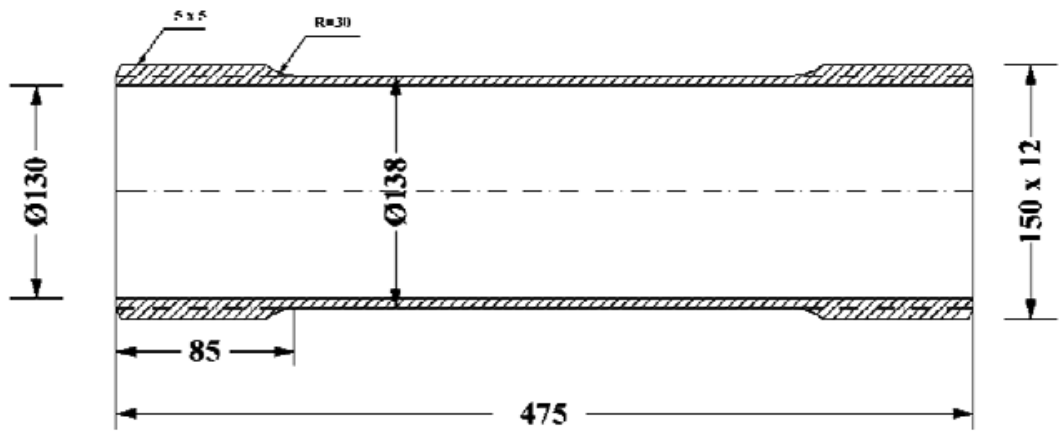

Fig.3. Tubular tube in glass/polyester composite. 


\subsection{Determination of the parameters $\beta$ and $a$}

From the resistance limit value $\sigma_{b}$ of the material (the value determined by the material breakdown testing), the loading values for the test in the long term are determined.

In our case, these values were taken respectively $0.9 \sigma_{b} ; 0.8 \sigma_{b} ; 0.7 \sigma_{b}$ and $0.6 \sigma_{b}$. For the studied material, $\sigma_{b}=98 \mathrm{MPa}$.

Expression (2.9) requires that testing should occur at both temporal intervals:

- Tests performed for $t_{*}<10$ hours

- Tests performed for $t_{*}>10$ hours.

For these tests, we use the stress standardized report $\frac{\sigma_{b i_{\bullet}}}{\sigma_{b}}$ :

The numerical values of the parameters $a$ and $\beta$ determined using experimental points in Fig.4 and expression (2.9) are given in Tab.1.

Table1. Values of parameters $a$ and $\beta$.

\begin{tabular}{|l|c|c|c|c|}
\hline \multirow{2}{*}{ Break time inter vals } & \multicolumn{2}{|c|}{$t *<10$ hours } & \multicolumn{2}{c|}{$t_{*}>10$ hours } \\
\cline { 2 - 5 } & $\beta$ & $a,\left(h^{\beta-1}\right)$ & $\beta$ & $a,\left(h^{\beta-1}\right)$ \\
\hline Values & 0.63 & 1.02 & 0.98 & 1.36 \\
\hline
\end{tabular}

Figure 4 shows the results of tests including the experimental points and curves approximation of the long term resistance for the two areas of longevity.

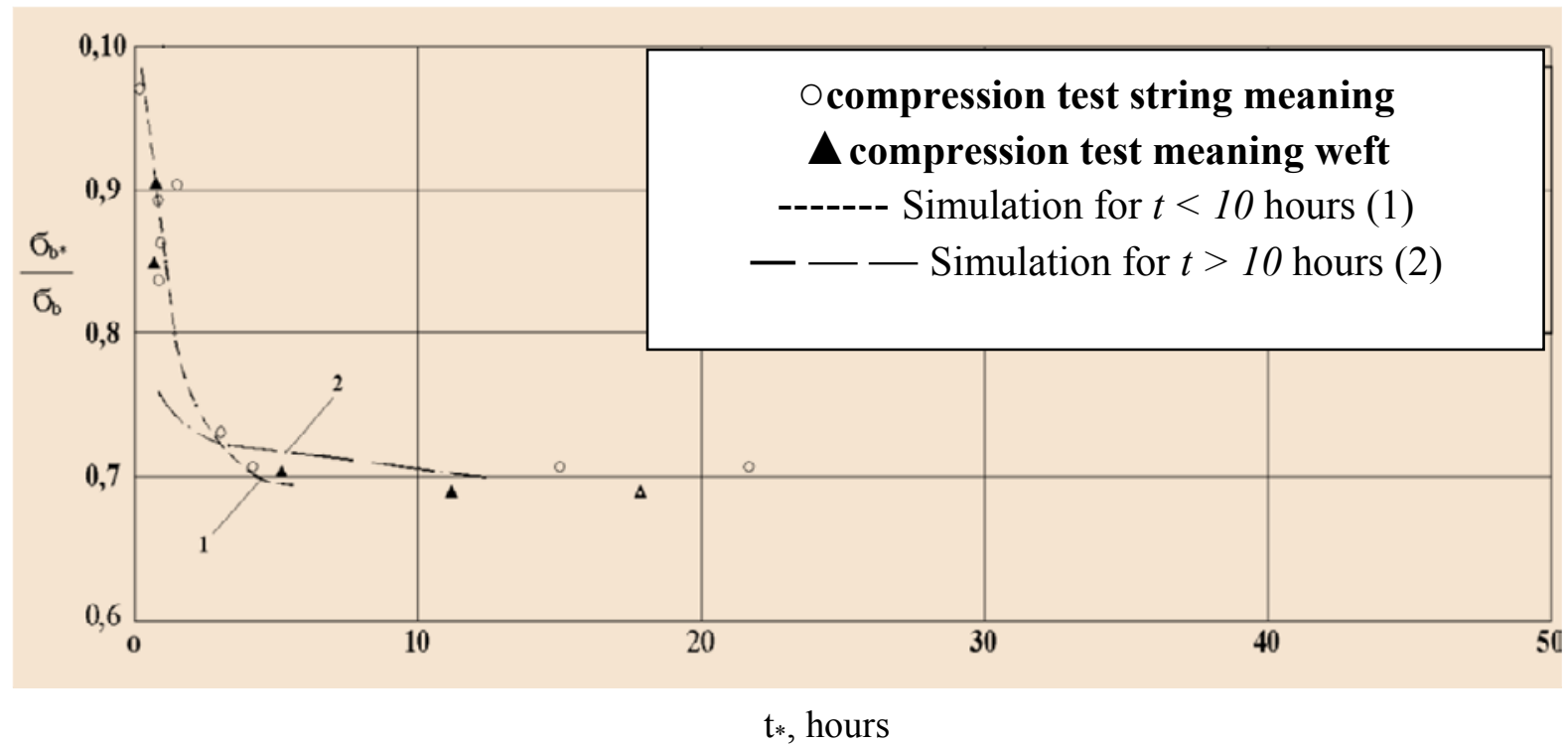

Fig.4. Experimental points and Abelian kernel approximation curves. 


\subsection{Explicit forms of heredity kernels and determination of life time and long term stress limit}

The values of the parameters determined allow use to establish explicit forms of heredity kernels. Thus, for the areas of major longevities we have

$$
\begin{aligned}
& \omega_{i k}(t-\xi)=\frac{0.0272 \prod_{i k}}{(t-\xi)^{\beta}}, \\
& \omega_{i k m n}\left(t-\xi_{1}, t-\xi_{2}\right)=\frac{7 \cdot 10^{-4} \prod_{i k m n}}{\left(t-\xi_{1}\right)^{\beta}\left(t-\xi_{2}\right)^{\beta}},
\end{aligned}
$$

and in this case, the life time will be

$$
t_{*}=\left[1.36\left(\prod_{i k} \sigma_{i k(0)}+\sqrt{\prod_{i k m n} \sigma_{i k(0)} \sigma_{m n(0)}}\right)\right]^{-50}
$$

here, the long-term stress limit

$$
\sigma_{b i *}=\frac{\sigma_{b}}{1,36 t_{*}^{0,02}}
$$

In the case of small longevities, we have

$$
\begin{aligned}
& \omega_{i k}(t-\xi)=\frac{0.444 \prod_{i k}}{(t-\xi)^{\beta}}, \\
& \omega_{i k m n}\left(t-\xi_{1}, t-\xi_{2}\right)=\frac{0.1971 \prod_{i k m n}}{\left(t-\xi_{1}\right)^{\beta}\left(t-\xi_{2}\right)^{\beta}}, \\
& t_{*}=\left[1.2\left(\prod_{i k} \sigma_{i k(0)}+\sqrt{\prod_{i k m n} \sigma_{i k(0)} \sigma_{m n(0)}}\right)\right]^{-2,7} .
\end{aligned}
$$

The long term stress limit will be

$$
\sigma_{b i *}=\frac{\sigma_{b}}{1,2 t_{*}^{0,37}}
$$

Curve (1) obtained in the field of small longevities gives a better approximation than curve (2), showing that the Abelian kernel which is a power law gives more satisfactory results for small periods of loading. However a number of studies related to long term test of polymeric composites show that in the case of long-term loading tests there is an exponential relationship between long-term strength and duration of loading of the material. Therefore the heredity kernel of the model must have an exponential form in the case of large longevities. 
Indeed, consider the exponential kernels of the form

$$
\begin{aligned}
& \omega_{i k}=b \prod_{i k} e^{-\alpha(t-\xi)}, \\
& \omega_{i k m n}=b^{2} \prod_{i k m n} e^{-\alpha\left(2 t-\xi_{1}-\xi_{2}\right)} .
\end{aligned}
$$

By integrating them in Eq.(2.1) with the same loading law, we obtain

$$
t_{*}=-\frac{1}{\alpha} \ln \left(1-\frac{\alpha}{b} \frac{\sigma_{b}}{\sigma_{b_{*}}}\right),
$$

which is the expression of the life time of the material using exponential kernel.

The long term stress limit would be

$$
\sigma_{b_{i *}}=\frac{\alpha \sigma_{b_{i}}}{b\left(1-e^{-\alpha t_{*}}\right)} .
$$

This last expression shows that when $t \rightarrow \infty, \sigma_{b i^{*}}$ tends towards a non-zero limit value. This is a considerable advantage because it means that it can be used to make predictions of the behaviour of the material for large domains of longevity. On the other hand expression (2.10) shows that when $t \rightarrow \infty, \sigma_{b i}{ }^{*}$ tends towards zero. One may infer that the Abelian type kernels are better suited for small longevities predictions as shown in experimental results (Fig.4).

The values of the parameters depend on the fields of longevity of the material. Asymptotic approximation curves boundaries define the value limits of the stresses to be taken into account for each type of loading.

On the other hand, the time needed for the rupture usually appears on the stress-rupture digraphs whose use is obvious. If one knows the stress, one reads life time.

Failure of many glass/polyester composites can occur earlier than expected and for small levels of stress. Therefore when it is a design of structures based on these materials, we must be sure that:

- The long term stress limit for the life time in the design is acceptable.

- Break loading time provided by the design exceeds the duration of intended use, so the material life.

The results of this work show that for the life time prediction of the polymeric glass fiber composites particular importance should be given on the establishment of the predictive model heredity kernels. This requires a correct experimental identification of the kernel parameters taking into account the expected operating life time.

\section{Conclusion}

This work presents:

1. The terms of the long-term behaviour tests that generate data required for the design calculations (engineering) and life time prediction, information unavailable in the technical literature. 
2. It is preceded to the study and analysis the heredity kernels. Could see that for studied material, these kernels are the product of static tensors of different orders by only the time-dependant functions.

3. Nonlinear viscoelastic model is identified by experimental determination of the viscoelastic parameters allowing establishing explicit forms of the heredity kernels.

4. It is highlighted the influence of these parameters therefore the heredity kernels on life time prediction of the composites. In particular, could see that Abelian kernels respond well to predictions from small periods of loading tests while for long loading time tests, exponential kernels are better suited for long term behaviour prediction of the material.

5. It is proposed formulas to determine the life time of the polymeric composites. These formulas are obtained for the Abelian and exponential kernels.

6. Are obtained the expressions for calculation the long term resistance limit of this material.

This proposed prediction method gives the possibility to establish behaviour of the material for long periods of operation and may be useful in engineering calculation for assessment the long term resistance of these materials.

\section{Nomenclature}

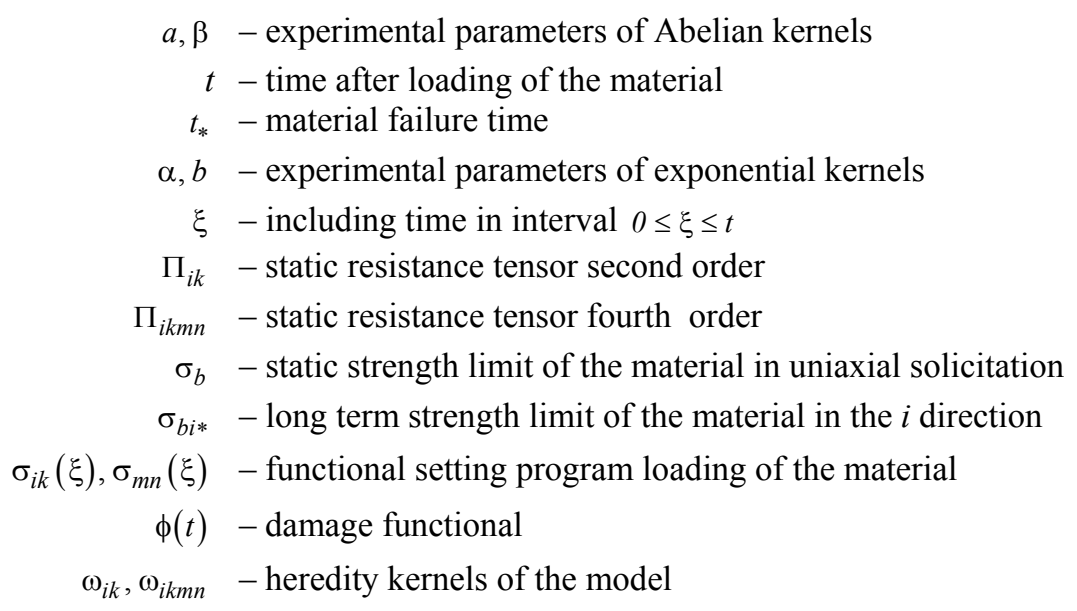

\section{References}

Augl J.M. (1987): Non linear creep effects of physical ageing, temperature and moisture of an epoxy resin. - J. Rheo., vol.31, pp.1-36.

Brinson H.F. and Tuttle M.E. (1986): Prediction of the long term creep compliance of general composite laminates. Exp. Mech., vol.26, pp.89-102.

Dimitrienko Yu. and Dimitrienko I. (1989): Long term strength of reinforced composites. - Mech. Comp. Mat., vol.25, pp.13-18.

Olodo E.T., Niang F., Adjovi E.C. and. Kopnov V.A. (2013): Long term behavior of composite material polyester-wood reinforced glass fiber. - Res. J. Appl. Sci. Eng. Tech., vol.6, No.2, pp.196-201

Pobedria B. (2007): On the effective behavior of nonlinear inelastic composites. - J. Mech. a Phys. Sol., vol.55, pp.1932-1963.

Pobedria B. (1983): On the theory of viscoelasticity of structurally inhomogeneous media. - J. Appl. Math. Mech., vol.47, pp.104-109. 
Schapery R.A. (2000): Nonlinear viscoelastic solids. - Int. J. Sol. Str., vol.37, pp.359-366.

Schapery R.A. (1997): Nonlinear viscoelastic and viscoplastic constitutive equations based on thermodynamics. Mech. Time-Dep. Mat., vol.1, pp.209-240.

Sullivan J.L. (1990): Creep and physical ageing of composites. - Comp. Sci. Tech., vol.39, pp.207-232.

Suvorova Y. (2010): Experimental and analytical methods for estimating durability of geosynthetic materials. - J. Mach. Man. Rel., vol.39, pp.391-395.

Zhang S.Y. and Xiang X.Y. (1992): Creep characterization of a fiber reinforced plastic material. - J. Rein. Pl. Comp., vol.2, pp.1187-1194.

Received: November 6, 2013

Revised: October 12, 2014 